\title{
Cost and logistics of alternative roll-out options for implementing human papillomavirus testing as a triage in cervical screening: results of the sentinel sites study
}

\author{
R Legood ${ }^{*,}$, Z Sadique', J Patnick ${ }^{2}$, H Kitchener ${ }^{3}$, R Kelly ${ }^{4}$ and S Moss ${ }^{4}$ \\ 'Department of Health Services Research and Policy, London School of Hygiene and Tropical Medicine, 15-17 Tavistock Place, London WCIH 9SH, UK; \\ ${ }^{2}$ NHS Cancer Screening Programmes, Sheffield, UK; ${ }^{3}$ Gynaecological Oncology Group, School of Cancer Studies and Enabling Sciences, University of \\ Manchester, Manchester Academic Health Science Centre, Manchester, UK; ${ }^{4}$ Centre for Cancer Prevention, Queen Mary University of London, Wolfson \\ Institute, Charterhouse Square, London ECIM 6BQ, UK
}

BACKGROUND: Previous studies have indicated that human papillomavirus (HPV) testing as a triage for managing equivocal cytology is cost-effective. The aim of this study was to assess the costs of alternative roll-out options.

METHODS: Detailed cost estimates were collected from six laboratories where HPV triage had been implemented. Costs were assessed for the two different service delivery models that were implemented; a 'hub and spoke model' of central HPV testing in a microbiology laboratory with separate cytology laboratories, and an 'integrated model' where HPV testing was conducted within the cytology laboratory.

RESULTS: Comparison of alternative delivery models indicated that setting up HPV processing within existing cytology laboratory, i.e., an 'integrated cytology/HPV laboratory' generated savings in staff time amounting to between $£ 2.54$ and 4.86 per sample processed. Running full HPV testing batches was also an important consideration. For full batches to be run on a twice weekly basis requires having no more than two laboratories per Strategic Health Authority.

CONCLUSIONS: To be cost-efficient, and to meet turn-around times, HPV testing needs to be conducted at integrated cytology/HPV testing centres with sufficient throughput to run full batches of HPV tests.

British Journal of Cancer (2012) I 107, 1574-1579. doi:I0.1038/bjc.2012.435 www.bjcancer.com

Published online 4 October 2012

(c) 2012 Cancer Research UK

Keywords: costs; human papillomavirus; cervical cancer

A number of economic analyses have been conducted that suggest that compared with repeat cytology it would be more cost-effective to manage women with low-grade cervical cytology results by utilising human papillomavirus (HPV) testing as a triage to refer women at higher risk for immediate colposcopy and returning HPV negative women to routine screening (Kim et al, 2002; Sherlaw-Johnson, Philipps, 2004; Legood et al, 2006). The implementation of HPV triage can be delivered in a number of different ways with HPV testing and cytology being performed in the same centre or samples sent to a central HPV testing centre based in a microbiology laboratory. To date, little research has been published on the optimal way to implement HPV testing within a screening programme.

The purpose of this study was to compare the costs and logistical implications of implementing HPV triage in a routine cervical screening programme (CSP) using data from the NHSCSP 'Sentinel Sites' study. A full description of this study and the epidemiological findings has been published previously (Kelly et al, 2011). Briefly six 'sentinel sites' were established to follow an agreed protocol for the use of HPV testing for women with borderline or mild dyskaryosis cytology results. At one site in

*Correspondence: Dr R Legood; E-mail: rosa.legood@|shtm.ac.uk Received 21 May 2012; revised 23 August 2012; accepted 30 August 2012; published online 4 October 2012
Bristol HPV testing was conducted within the cytology laboratory. At Manchester HPV testing was conducted off-site at a microbiology laboratory. Four more sites: Liverpool, Manchester, Northwick Park and Sheffield were spoke sites sending the relevant cytology samples for HPV testing at the other two sites. Together these six sites represent $\sim 10 \%$ of the English Screening Programme. All women aged 25-64 with routine cytology reported as borderline or mild dyskaryosis were eligible for inclusion in the study. Women who tested negative for HPV were returned to routine recall at 3 or 5 years depending on their age, and no further information was collected; women who tested positive were referred for a colposcopic examination. This study reports on the detailed resource and costing data on implementation and running costs that were collected alongside the project.

\section{MATERIALS AND METHODS}

For the purpose of costing two alternative service delivery models for HPV testing were evaluated: a 'hub and spoke model', and an 'integrated HPV/cytology laboratory' (Figure 1). In the 'hub and spoke model', cytology laboratories without HPV testing facilities onsite, were considered as spokes. In line with the protocol, cytology samples that indicated mild or borderline results required HPV testing. With the hub and spoke model the relevant cytology samples were transported to a central microbiology laboratory 


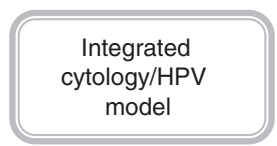

Hub and spoke delivery model
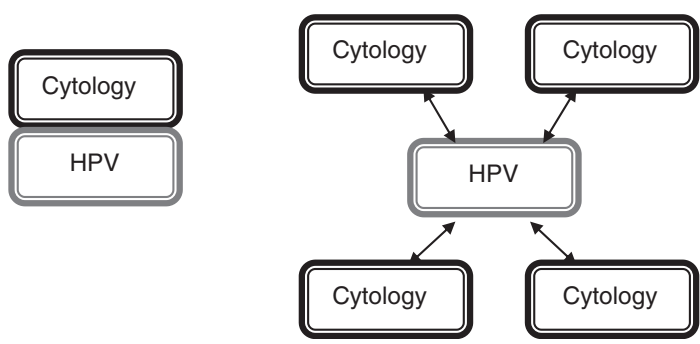

Figure I Alternative delivery models.

(the hub) for HPV testing. In contrast, with the integrated HPV laboratory both cytology and HPV testing facilities are provided at the same centre. Costs were estimated for both of the delivery models. We present separately the costs of implementation and the running costs.

The cost analysis was carried out from the NHS perspective. All costs refer to 2009; where required costs were adjusted using the Hospital and Community Health Service pay and price index (Curtis 2010). Equipment and consumable costs associated with HPV testing were estimated from the cost incurred at laboratories and in consultation with suppliers based on 5 year contract for leasing. Unit costs of staff time were derived from observational studies, mostly undertaken specifically for the 'Sentinel Sites' study, and from existing tariffs and contracts, as well as from published sources. Salary costs were then attributed for each activity based on the mid-scale point for the corresponding band in the Agenda for Change salary structure (Pay Circular (A for C) 1/2006, 2006, (see Appendix Tables A1 and A2). Salary costs included qualifications and NHS employers' costs (i.e, the employer's national insurance contribution plus $14 \%$ of salary for employer's contribution to superannuation).

Three separate categories of cost were considered: (a) training and implementation costs (b) cost of administration, identifying samples/specimen reception and transport (c) the cost of HPV testing.

\section{Training and implementation}

A cost questionnaire was devised to assess the potential additional costs of HPV testing for the 'Sentinel Site' laboratories not performing HPV testing, the spoke laboratories. The questionnaire assessed the following components of cost: training costs for cytology laboratory staff, training cost for staff taking cytology samples, cost of transporting liquid-based cytology samples for HPV testing and administrative and other costs such as Information Technology (IT) system change.

A cost questionnaire was also devised to identify the cost incurred at HPV testing laboratories, which included staff training costs, equipment and consumable costs. The questionnaire identified the costs of training current staff for performing HPV testing. As these training and implementation costs are a one-off cost they are not included in subsequent cost analysis.

\section{Cost of administration, identifying samples/specimen reception and transport}

In the 'hub and spoke' model, costs of administration and identifying/specimen reception are incurred in both spoke laboratories (i.e., cytology laboratories) and the hub laboratory
(HPV laboratory). Record sheets were developed to record the amount of staff activity related to HPV testing in spoke laboratories. Timings were recorded for the following activities: identifying cytological samples for HPV testing, packing samples for transporting to the HPV laboratory, updating clinical records and administration time. Confidence intervals were estimated for the timings assuming a normal distribution. Timings were multiplied by cost weights for the type of staff performing each activity as shown in the Appendix.

The slides were then transported to HPV laboratories. The costs of spoke-to-hub transport of vials for processing were estimated from the average journey transport costs and numbers of samples transported. Record sheets were developed to record the amount of staff activity related to HPV testing in a hub laboratory. The costs of following staff activities were identified: specimen reception and unpacking, updating clinical records and administration time.

In the integrated 'HPV/cytology' laboratory scenario there is no requirement for transportation costs and the following costs were included identifying cytological samples for HPV testing, updating clinical records and administration time. Data from the record sheet survey were used to determine the duration of time required and grades of staff undertaking each activity and costed as described above.

\section{Costs of performing HPV tests}

The cost of HPV testing includes equipment, consumables and staff costs. Equipment and consumables for HPV testing were based on the Qiagen HC2 (Crawley, England, UK) as used in the 'Sentinel Sites' study. A range of further HPV test alternatives are currently on the market and further ongoing evaluation is being undertaken on these tests. Potential differences could include differences in price and batch sizes required to achieve efficiency and avoid waste.

Costs were based on a manual preparation system as used in 'Sentinel site' laboratories undertaking the HPV testing. Again further equipment is available from other manufacturers with elements of automation, but this was not assessed within this evaluation. Costing of HPV equipment/consumables was based on 5 year contract lease prices and the modelled throughput of different sized HPV laboratories. Indicative prices for equipment and maintenance were obtained in confidence from the manufacturer. To maintain confidentiality over indicative prices, we present prices in combination with the staff costs associated with preparing HPV tests.

A questionnaire was devised and sent to the laboratories undertaking HPV testing to assess the costs of other equipment not provided by the manufacturer and to identify the consumable and staff time involved. Information was also obtained on the costs of HPV consumables and staff processing time depending on the number of tests being processed in each batch. Costs of HPV testing are highly dependent on the batch size being processed at one time, and costs are therefore presented for batches of different sizes.

During the 'Sentinel Sites' study, HPV tests were processed on a twice weekly basis. An important consideration for HPV testing is that it can be integrated within the overall cytology pathway to meet government targets of women receiving their results within 14 days of a smear being taken (Department of Health, 2007). Within the 'Sentinel Sites' study - some cytology laboratories successfully managed to achieve a 14-day-turn-around time including sending smears for HPV testing.

An alternative option would be that HPV processing was conducted on a daily basis to further improve turn-around times; however, this would only be economically efficient if there were a sufficient quantity of tests. We assessed the volume of HPV tests for each of these roll-out options by combining the data collected for the epidemiological evaluation on the volume of HPV tests (for both HPV triage of mild and borderlines and HPV test of cure) 
with estimates of the numbers of routine cytology tests from the; the national screening programmes statistical returns (KC-61).

\section{RESULTS}

\section{Training and implementation}

A range of staff were involved with training for handling HPV tests that would then be sent for processing at a hub HPV testing laboratory. Training topics covered for identifying samples included following protocols and correct placement of slide numbers. Staff involved with packing cytology samples were given training on: explaining protocols, quality control steps to ensure correct totals were included and health and safety guidance. Training on updating records with HPV results included verbal explanation of protocols, instruction in the use of databases and quality control steps to reduce transposition errors. Overall training times for cytology staff varied from about an hour to half a day, and were mainly provided in-house. It is likely that these training costs for cytology staff would be similar for either type of delivery model.

The number of staff who received training about HPV testing provided by the cytology laboratories varied across the sites, from 52 to 212 . Where fewer staff were trained, strategies were used such as cascading information to staff and distributing HPV information packs. An alternative model was to organise two formal presentations for all cervical sample takers lasting $3 \mathrm{~h}$, and then to visit each general practitioner practice where there had been no representation at either event. The cost of this training varied between no direct costs (apart from staff time) to $£ 2500$. It is anticipated that these training costs should be covered locally as part of routine training updates for sample takers.

In the laboratory where HPV testing was already being undertaken, training of staff on HPV testing was carried out through in-house training. In the integrated HPV/cytology laboratory, training was carried out by both the manufacturer and in-house staff. The manufacturer provided training on running assays and occasional troubleshooting matters. In-house training was also provided for staff on receiving and preparing samples, and reporting results.

There were some IT system changes in all the cytology laboratories for changing codes, setting up the system and establishing electronic links for the Exeter system. Each site had to ensure that new result codes matched with the final result letters sent to women. On average it took 3 days to perform IT changes. Most sites did not incur any financial costs (beyond in house staff time), other than one site that spent $£ 2250$ on IT system changes.

\section{Staff time for administration, identifying samples/ specimen reception}

Table 1 presents the time incurred for staff activity. Data were collected at four out of the five sites where HPV testing was conducted offsite (spokes). Timings were recorded for the following activities: identifying cytological samples for HPV testing, packing samples for transporting to the HPV laboratory, updating clinical records and administration time. These data show very similar results across three of the four sites, with the total staff time per HPV sample at between 5 and 6 min (Table 1), at one site (Site $\mathrm{C}$ ) the timings were significantly higher.

In the integrated cytology and HPV testing laboratory, only the times for identifying samples for HPV testing and administration for reporting the results were recorded (Table 2). At the hub HPV processing centre hub (Manchester), we considered the costs of specimen reception and administration including reporting results back to the cytology laboratory (Table 3). Our data indicated that the administration times were slightly lower at the hub HPV
Table I Time per HPV sample in cytology laboratories with HPV testing offsite in seconds - mean $(95 \% \mathrm{Cl})$

\begin{tabular}{lcccc}
\hline Activity & \multicolumn{1}{c}{ A } & \multicolumn{1}{c}{ B } & C & D \\
\hline Identify sample & $126(50,202)$ & $45(33,57)$ & $44(28,60)$ & $70(45,96)$ \\
Packing & $96(56,136)$ & $58(52,64)$ & $182(130,235)$ & $67(14,119)$ \\
Update records & $76(39,113)$ & $9 \mid(79,103)$ & $223(181,266)$ & $175(128,220)$ \\
Admin time & $28(7,49)$ & $|3|(\mid 17,146)$ & $338(165,5 \mid 1)$ & $0(0,0)$ \\
Total & $326(153,500)$ & $325(280,370)$ & $787(502,107 \mid)$ & $31 \mid(\mid 87,436)$ \\
\hline
\end{tabular}

Abbreviations: $\mathrm{Cl}=$ confidence interval; HPV = human papillomavirus. A-D represent timings at different laboratories $-n=6$ batches at each site.

Table 2 Time per sample in HPV laboratories in seconds-mean $(95 \% \mathrm{Cl})$

\begin{tabular}{lcc}
\hline Activity & $\begin{array}{l}\text { Hub HPV } \\
\text { testing centre }\end{array}$ & $\begin{array}{l}\text { Integrated cytologyl } \\
\text { HPV laboratory }\end{array}$ \\
\hline $\begin{array}{l}\text { Specimen reception (separate) } \\
\text { Identifying samples (integrated) }\end{array}$ & $62(56,68)$ & $197(123,27 \mid)$ \\
$\begin{array}{l}\text { Reporting results administration } \\
\text { Total }\end{array}$ & $50(26,76)$ & $58(28,77)$ \\
\hline
\end{tabular}

Abbreviations: $\mathrm{Cl}=$ confidence interval; HPV = human papillomavirus.

Table 3 Cost per sample-mean (95\% Cl) (range)

\begin{tabular}{|c|c|c|}
\hline Activity & $\begin{array}{l}\text { Hub HPV } \\
\text { testing centre } \\
(£)\end{array}$ & $\begin{array}{l}\text { Integrated HPVI } \\
\text { cytology laboratory } \\
\text { (f) }\end{array}$ \\
\hline $\begin{array}{l}\text { Cost of staff time in cytology } \\
\text { laboratory (excluding laboratory } \\
\text { C) }\end{array}$ & $\begin{array}{l}2.66(1.72,3.61 ; \\
2.28(1.56,2.99))\end{array}$ & NA \\
\hline Transport cost & & NA \\
\hline $\begin{array}{l}\text { Transport distance- }-(150- \\
300 \mathrm{~km}) \text {, }\end{array}$ & $2.76(2.20,3.33)$ & \\
\hline Transport distance- $-(<50 \mathrm{~km})$ & $0.44(0.38,0.50)$ & \\
\hline Specimen reception (separate) & $0.24(0.22,0.26)$ & - \\
\hline Identifying samples (integrated) & - & $0.76(0.48,1.05)$ \\
\hline Reporting results & $0.24(0.12,0.36)$ & $0.28(0.18,0.37)$ \\
\hline \multicolumn{3}{|l|}{ Total } \\
\hline $\begin{array}{l}\text { Transport distance-_-(150- } \\
300 \text { km) }\end{array}$ & $5.90(5.20,7.56)$ & \\
\hline Transport distance- $-(<50 \mathrm{~km})$ & $3.58(3.38,4.73)$ & $1.04(0.66,1.42)$ \\
\hline
\end{tabular}

Abbreviations: $\mathrm{Cl}=$ confidence interval; $\mathrm{HPV}=$ human papillomavirus; $\mathrm{NA}=$ not applicable.

testing centre, just under 2 min compared with $4.5 \mathrm{~min}$ at the 'integrated HPV/cytology laboratory' where the relevant samples for HPV testing had to be identified. However, as specimens had to be identified in the hub and spoke delivery model as shown in Table 1, overall the combined staff time involved was much greater for the 'hub and spoke' delivery model compared with the integrated model.

\section{Cost comparison between 'hub and spoke model' and 'integrated cytology/HPV laboratory': administration, identifying samples/specimen reception and transport}

Costs of the hub and spoke model were more expensive in part because of transport costs. Each centre sent samples for HPV testing to HPV laboratories twice a week. The average number of samples sent per journey was 14 (range 10-18). The cost per journey across centres varied from $£ 5$ to 50 . Sentinel sites A and D were located at relatively close proximities to HPV laboratories 
(just under $50 \mathrm{~km})$ compared with sites B and C (150-300 km). Sites A and D both incurred similar delivery costs - transporting up to 12 samples at a price of $£ 5$ per box and a larger box cost $£ 25$ to transport up to 40 samples. Sites B and C generally sent samples in a larger box that could contain 40 or more HPV samples at a cost of between $£ 40$ and 50 per box. The average transport costs per sample across the two sites located $\sim 50 \mathrm{~km}$ away were $£ 0.44$ (range $£ 0.38$ to 0.50 ). For those sites located further from the HPV processing laboratory $(150-300 \mathrm{~km})$, average transport costs per sample were higher at $£ 2.76$ (range $£ 2.20-3.33$; see Appendix Table $\mathrm{A} 3)$.

The 'integrated HPV/Cytology' laboratory leads to lower total staff costs for administration, identifying samples/specimen reception than that of 'hub-and-spoke' model. The total staff administrative costs for the integrated laboratory were $£ 1.04$ compared with $£ 3.14$ for the integrated model. We also calculated the total staff cost excluding laboratory $\mathrm{C}$ where the timings were much higher than the other spoke laboratories and this lowered the total cost to $£ 2.76$.

Overall, with the 'hub-and-spoke' model staff and transportation costs ranged from $£ 3.58$ to 5.90 per sample depending on the transportation distance, the equivalent costs for the integrated laboratory were $£ 1.04$ (See Table 3 ). The integrated laboratory does not incur any cost for transporting samples.

\section{Costs of performing HPV testing}

During the Sentinel sites project the Qiagen HC2 test was used. Each kit has 96 wells including 8 wells that are used as controls for each batch. There are economies of scale related to running full batches including: the need for eight controls even with smaller batches, use of slightly more consumable costs per test, higher average equipment costs and increases in staff time per test. The cost of HPV test varied from $£ 12.83$ to 20.97 depending on the batch size. The cost of an HPV test includes the cost of equipment, consumables, maintenance and staff time. These costs all increase when running smaller batches. It is most cost-efficient to perform HPV testing at full batch capacity (88 samples per batch) $£ 12.83$ per test. However, the marginal increase in cost for running 66 samples compared with 88 is only an additional $£ 1.20$ per test, which if HPV testing was operating in a greater number of laboratories that do not have sufficient capacity to run full batches could potentially be offset by decreased transport costs.

The volume of tests for HPV processing centres serving different sized cytology laboratories were estimated from the 'Sentinel Site' study data. Options included as follows:

- HPV testing in individual cytology laboratories (35 000 cytology slides per year).
- HPV testing across cytology laboratories (100 000 cytology slides per year).

- Two HPV laboratories at Strategic Health Authority (SHA) level.

- One HPV laboratory at SHA level.

- One National HPV processing laboratory.

Table 4 shows that running HPV testing daily in most of the options would be inefficient as there would be unused capacity. Running daily HPV testing with full batches would only be possible if an HPV processing centre serves cytology laboratories reading over 0.3 million slides per year.

On the basis of running HPV tests twice a week it is possible to run full batches with two HPV testing centres per SHA. This may also be a cost-efficient option if transport costs are reduced, especially where the SHA covers a large geographical area. However, it should be noted that the HPV testing workload figures will vary from according to the protocols being followed.

\section{DISCUSSION}

Although there have been a number of cost-effectiveness analyses conducted assessing the overall cost-effectiveness of implementing HPV triage (Kim et al, 2002; Sherlaw-Johnson and Philipps, 2004; Legood et al, 2006), we did not identify any previous detailed costing analyses of the cost-efficiency of alternative roll-out options. We found that the main implementation cost component for the cytology laboratories was making changes to the IT system, although no costs were substantive. Comparison of alternative delivery models indicated that by setting up HPV processing within the existing cytology laboratory, in an integrated cytology/ HPV laboratory' generated savings in staff time ranging from between $£ 2.54$ and 4.86 . The additional costs incurred by having a 'hub and spoke model' with cytology laboratories sending samples to another site for processing included the cost of transportation and extra administrative workload.

Our estimate for the cost of HPV testing including the staff, consumable and equipment cost ranged from $£ 12.83$ to 20.97 depending on the batch size. To maintain confidentiality over prices we did not present disaggregated costs. However, our result for running full batches $£ 12.83$ is only slightly higher than an estimate from the ARTISTIC study for full batches of £10.57-10.38 (Kitchener et al, 2009).

When considering alternative implementation options a key issue is the cost of HPV testing that increase when running smaller batches if the Hybrid Capture II system is used. Overall, our results indicate that with two testing centres per SHA it would be possible to have full batch sizes, given a twice weekly testing schedule and this would be consistent with government targets for a

Table 4 Volume of HPV tests for alternative roll-out options ( $\mathrm{Cl}$ ) and costs (range)

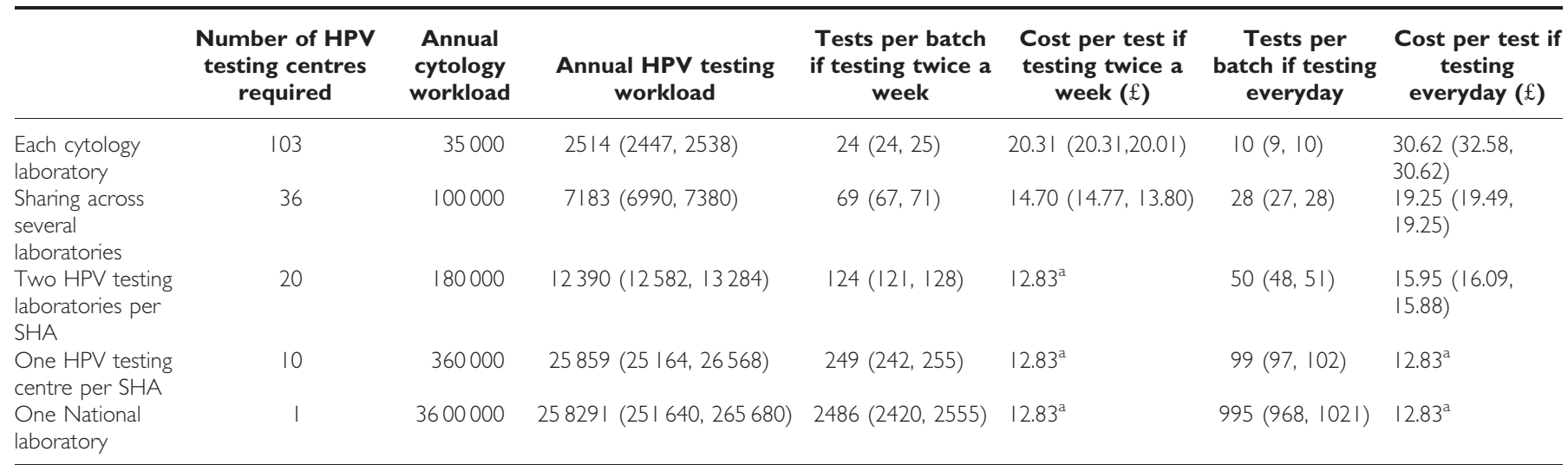

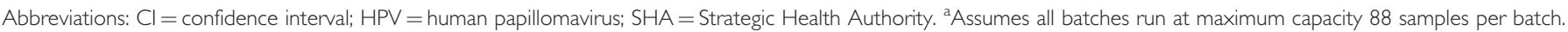


14-day-turn-around (Department of Health, 2007). Keeping within the 14-day-turn-around time is important for ensuring there is not disutility for women waiting for results. Given that the cost per HPV test with this batch size is only an extra $£ 1.20$, this may also be a cost-efficient option if transport costs are reduced, especially where the SHA covers a large geographical area. Although Hybrid Capture II was used within this evaluation, alternative options that require smaller batch sizes are also available and these have not been evaluated within this study.

A limitation of our analysis is that we have not evaluated the cost of building space and utilities so in practice the total costs may be higher. Our estimates of the volumes of HPV samples to be processed are based on mean HPV positivity rates for mild and borderline cytology results. In practice as shown in the Sentinel Site study, (Kelly et al, 2011) there will be some variations locally depending with some place having higher HPV positivity rates and therefore higher-throughput volumes and others having lower rates and throughput volumes.

An alternative approach would be to have one national laboratory or only a few very large laboratories. A further advantage of processing HPV tests at higher volumes is the potential to generate savings from economies of scale. For example, further HPV equipment could be used that increases the automation of the HPV testing process. The results of this project suggest that transport costs were significantly higher over longer distances, although it is possible that lower and more efficient transport options could be found such as transferring samples to a central point before sending on. Primary HPV testing, which is likely to be on the future policy agenda, would generate many more samples and thus make it possible to achieve economies of scale within a smaller screened area.

\section{ACKNOWLEDGEMENTS}

The study was funded by the NHS Cancer Screening Programme. We thank members of the HPV Special Interest Group for their helpful comments and inputs: R Albrow: School of Cancer Studies and Enabling Sciences, University of Manchester, Manchester Academic Health Science Centre, Manchester, UK; A Bailey and A Sargent: HPA Manchester, Manchester, UK; H Cubie and C Moore: Scottish HPV Reference Laboratory, Edinburgh, UK; K Denton and K Hunt: Southmead Hospital, Bristol, UK; M Desai and J Marshall: Central Manchester University Hospitals NHS Foundation Trust, Manchester, UK; K Ellis, JHF Smith and J Tidy: Sheffield Teaching Hospitals NHS Foundation Trust, Sheffield, UK; C Evans, T Giles and L Turnbull: Royal Liverpool University Hospital, Liverpool, UK; M Ferry and T Levine: North West London Hospitals NHS Trust, Harrow, UK; A Fisher: Queen Elizabeth Hospital, Gateshead, UK; V Frew and R Lonsdale: Norfolk and Norwich University Hospital, Norwich, UK; R Howell-Jones and K Soldan: Health Protection Agency, London, UK; P Muir: HPA South West Regional Laboratory, Bristol, UK; A Turner: Manchester Royal Infirmary, Manchester, UK; J Rimmer and R Winder: NHS Cancer Screening Programmes, Sheffield, UK; P Walker and M Young: Royal Free Hospital, London, UK; J Medlock: University Hospital Aintree, Liverpool, UK.

\section{REFERENCES}

Curtis L (2010) Unit Cost of Health and Social Care: Personal Social Services Research Unit. University of Kent. http://www.pssru.ac.uk/pdf/ uc/uc2010/uc2010.pdf

Department of Health Cancer Reform Strategy (2007)

Kelly RS, Patnick J, Kitchener HC, Moss SM, NHSCSP HPV Special Interest Group (2011) HPV testing as a triage for borderline or mild dyskaryosis on cervical cytology: results from the Sentinel Sites study. $\mathrm{Br}$ J Cancer 105(7): 983-988

Kim JJ, Wright TC, Goldie SJ (2002) Cost-effectiveness of alternative triage strategies for atypical squamous cells of undetermined significance. JAMA 287(18): 2382-2390

Kitchener HC, Almonte M, Gilham C, Dowie R, Stoykova B, Sargent A, Roberts C, Desai M, Peto J, ARTISTIC Trial Study Group (2009)

ARTISTIC: a randomised trial of human papillomavirus (HPV) testing in primary cervical screening. Health Technol Assess 51: 1-150

Legood R, Gray A, Wolstenholme J, Moss S (2006) Lifetime effects, costs, and cost effectiveness of testing for human papillomavirus to manage low grade cytological abnormalities: results of the NHS pilot studies. BMJ 332(7533): 79-85

Pay Circular (A for C) 1/2006 (2006) Pay and Conditions for NHS staff covered by the Agenda for Change agreement. In: Employers $\mathrm{N}$ (ed.). http://www.nhsemployers.org/Aboutus/Publications/PayCirculars/Pages/ PayCircularAfC12006.aspx

Sherlaw-Johnson C, Philipps Z (2004) An evaluation of liquid-based cytology and human papillomavirus testing within the UK cervical cancer screening programme. Br J Cancer 91(1): 84-91

This work is published under the standard license to publish agreement. After 12 months the work will become freely available and the license terms will switch to a Creative Commons Attribution-NonCommercial-Share Alike 3.0 Unported License.

\section{APPENDIX}

Table Al Cost per minute for laboratory staff

\begin{tabular}{lcc}
\hline Staff & Cost per min $(£)$ & Source \\
\hline MLA & 0.19 & $\begin{array}{c}\text { Curtis 2010; Pay Circular } \\
(\text { A for C) } 1 / 2006\end{array}$ \\
MLA3 & 0.20 & $\begin{array}{c}\text { Curtis 2010; Pay Circular } \\
\text { (A for C) } 1 / 2006\end{array}$ \\
Cytoscreener & 0.24 & $\begin{array}{c}\text { Curtis 2010; Pay Circular } \\
\text { (A for C) } 1 / 2006\end{array}$ \\
BMS & 0.29 & $\begin{array}{c}\text { Curtis 2010; Pay Circular } \\
\text { (A for C) } 1 / 2006\end{array}$ \\
Senior BMS & 0.42 & $\begin{array}{c}\text { Curtis 2010; Pay Circular } \\
\text { (A for C) } 1 / 2006\end{array}$
\end{tabular}

Table Al (Continued)

\begin{tabular}{|c|c|c|}
\hline Staff & Cost per $\min (£)$ & Source \\
\hline Pathologist & 1.36 & Kitchener et al, 2009 \\
\hline Advanced practitioner & 1.10 & $\begin{array}{l}\text { Curtis 2010; Pay Circular } \\
\text { (A for C) } 1 / 2006\end{array}$ \\
\hline Consultant & 2.82 & $\begin{array}{c}\text { Curtis 2010; Pay Circular } \\
\text { (A for C) } 1 / 2006\end{array}$ \\
\hline
\end{tabular}

Abbreviations: $\mathrm{BMS}=$ biomedical scientist; $\mathrm{MLA}=$ Medical Laboratory Assistant. 
Costs and logistics of the sentinel site study

$\mathrm{R}$ Legood et al

Table A2 Activities undertaken by staff type

\begin{tabular}{|c|c|c|c|c|c|}
\hline & $\begin{array}{c}\text { Cyto screener } \\
\text { (\%) }\end{array}$ & $\begin{array}{l}\text { BMS } \\
(\%)\end{array}$ & $\begin{array}{c}\text { Senior BMS } \\
(\%)\end{array}$ & $\begin{array}{l}\text { MLA } \\
(\%)\end{array}$ & $\begin{array}{c}\text { MLA3 } \\
(\%)\end{array}$ \\
\hline $\begin{array}{l}\text { Identify } \\
\text { sample }\end{array}$ & - & 33 & 50 & 17 & - \\
\hline Packing & 24 & 48 & 28 & - & - \\
\hline $\begin{array}{l}\text { Update } \\
\text { records }\end{array}$ & - & 46 & 27 & - & 27 \\
\hline Admin time & 8 & 68 & 24 & - & - \\
\hline
\end{tabular}

Total costs of staff activities were calculated by multiplying the staff time involved by a weighted cost based on different staff undertaking each activities. Confidence intervals for staff costs were generated by using these upper and lower confidence intervals for staff time.
Table A3 Transport cost

\begin{tabular}{|c|c|c|c|c|}
\hline Site & $\mathbf{A}$ & B & C & D \\
\hline $\begin{array}{l}\text { How many times a week } \\
\text { are LBC samples sent to } \\
\text { HPV test centre? }\end{array}$ & 2 & 2 & 2 & 2 \\
\hline Cost per journey & $\begin{array}{l}£ 5 \text { for } 10-16 \\
\text { samples } £ 25 \\
\text { for } 40+ \\
\text { samples }\end{array}$ & $£ 40+$ packaging & $£ 50$ & $\begin{array}{l}£ 5 \text { for } 12 \\
\text { samples in } \\
\text { a box }\end{array}$ \\
\hline $\begin{array}{l}\text { On average how many } \\
\text { samples are sent each } \\
\text { journey }\end{array}$ & 10 & 18 & 15 & 13 \\
\hline $\begin{array}{l}\text { Distance from HPV testing } \\
\text { laboratory in } \mathrm{km}\end{array}$ & 49 & 293 & 158 & 47 \\
\hline $\begin{array}{l}\text { What is the maximum } \\
\text { number of samples that } \\
\text { can be sent at a time for } \\
\text { the same price? }\end{array}$ & $\sim 200$ for $£ 25$ & NA & $\begin{array}{l}\text { Up to } \\
\text { I kg in } \\
\text { weight }\end{array}$ & $\begin{array}{l}12 \\
\text { centrifuge } \\
\text { tubes max } \\
\text { per box }\end{array}$ \\
\hline Average transport cost & $£ 0.50$ & $£ 2.20$ & $£ 3.33$ & $£ 0.38$ \\
\hline
\end{tabular}

Abbreviations: HPV = human papillomavirus; LBC = liquid-based cytology. 